\title{
Ciclo de Mejora en el aula de Matemáticas
}

\section{Improvement Cycle in Classroom of Mathematics}

INMACULADA CONCEPCIÓN MASERO MORENO

ORCID ID: https://orcid.org/0000-0002-4023-8916 Universidad de Sevilla

Departamento de Economía Aplicada III imasero@us.es

Fecha recepción:

Fecha aceptación:

DOI: http://dx.doi.org/10.12795/9788447221912.104

Pp.: 2337-2355 


\section{Resumen}

En este trabajo se presentan los resultados de un ciclo de mejora en el aula (CIMA) en la asignatura cuantitativa Matemáticas. Se expone el mapa de contenido, dos actividades de control y un cuestionario inicial-final. Los resultados muestran que esta propuesta influye positivamente en el aprendizaje.

Palabras clave: Matemáticas, Doble Grado en Finanzas y Contabilidad y Relaciones Laborales y Recursos Humanos, docencia universitaria, experimentación en docencia universitaria, Ciclo de Mejora en el Aula (CIMA).

\section{Abstract}

This paper presents the results of the Improvement cycle in Classroom (ICIC) of the quantitative subject Mathematics. We expose the map of contents, two activities of control and the initial-final questionnaire. The results show that this proposal have a positive influence in the learning process.

Keywords: Mathematics, Doble Grado en Finanzas y Contabilidad y Relaciones Laborales y Recursos Humanos, University Teaching, University teaching experimentation, Improvement Cycles in Classroom (ICIC).

Jornadas de Formación e Innovación Docente del Profesorado | № 2 (2019) Esta obra se distribuye con la licencia Creative Commons Reconocimiento-NoComercial-SinObraDerivada 4.0 Internacional (CC BY-NC-ND 4.0.) 


\section{Contexto}

Durante los cursos 2017/18 y 2018/19 he realizado ciclos de mejora en el aula (CIMA) en la asignatura Matemáticas de primer curso del Doble Grado en Finanzas y Contabilidad y Relaciones Laborales y Recursos Humanos de la Universidad de Sevilla. Es una asignatura de formación básica de 6 créditos que se imparte en el primer cuatrimestre.

En el curso pasado se introdujeron diversas modificaciones en el programa de la asignatura que han tenido su continuidad en el presente curso. Esto ha hecho que se haya reorganizado el temario y la ubicación de determinados contenidos. Por este motivo, me ha parecido conveniente retomar el ciclo del curso 17/18 y adecuarlo a la estructura que adquiere el tema en la nueva propuesta de programación de la asignatura.

Desde este nuevo programa de contenido de la asignatura, propongo un nuevo ciclo de 10 horas de duración en el que se aborda el Análisis de las funciones reales de varias variables reales. En esta parte del temario se pone de manifiesto como esta asignatura proporciona herramientas matemáticas para estudiar y analizar conceptos económicos como la marginalidad y la elasticidad.

Al igual que en los años anteriores, la asignatura tiene un único grupo. La docencia se imparte dos días a la semana en bloques de dos horas, lunes y martes por la tarde. En el presente curso, el aula tiene capacidad para 60 estudiantes. El espacio está organizado en dos columnas y seis filas (10 alumnos por fila, en la primera columna 6 y en la segunda 4). Las mesas son dobles, es decir, dos estudiantes comparten mesa. No están fijadas al suelo, lo que facilita la movilidad a la hora de reorganizar la clase para introducir dinámicas de trabajo en grupo. Esto también me facilita a mí el acercamiento a todos los estudiantes, ya que puedo desplazarme con facilidad entre las filas. 
Otra característica importante del aula es la ubicación de la pantalla en la que se proyectan los materiales de apoyo que preparo para cada clase o las pruebas que realizamos on line durante el trabajo presencial. La pantalla no oculta la amplia pizarra de la que dispone el aula, por lo que puede estar proyectado algún resultado teórico que nos interese tener presente y al mismo tiempo estar trabajando con los estudiantes en la pizarra.

En este curso, las características de este escenario lo hacen apropiado para poder trabajar con la metodología elegida y mejorar las relaciones estudiante-grupo-docente.

Como puede observarse, las metodologías de enseñanza-aprendizaje se han elegido teniendo en cuenta el escenario y el contexto disciplinar de los contenidos (De Miguel, 2009).

Actualmente, hay 63 estudiantes matriculados que, mayoritariamente, han cursado el Bachillerato de Ciencias Sociales.

\section{Diseño previo del Ciclo de Mejora en el Aula (CIMA)}

En esta asignatura es primordial poner de manifiesto la relación entre los conceptos y fenómenos económicos y los conceptos y herramientas matemáticas del Cálculo Diferencial e Integral. Sin embargo, no podemos olvidar que para poder aplicar estos conceptos y herramientas en el contexto económico, empresarial y financiero es necesario que el estudiante tenga un dominio adecuado de las funciones, el cálculo e interpretación de las derivadas parciales y la aproximación de las funciones a través de polinomios. Esta parte del temario es nueva para el alumnado, y supone una extensión del conocimiento que ya tiene de las funciones reales de variable real. 


\section{Principios didácticos}

Para un planteamiento adecuado del ciclo, he elaborado un cuestionario relación al conocimiento matemático básico y previo del alumnado. Dicho cuestionario me proporciona datos que me permiten plantear la enseñanza de forma acorde al nivel y amplitud de su conocimiento matemático. Este cuestionario es el instrumento que me permite realizar una evaluación inicial que, además, me proporciona información sobre sus expectativas en relación a la asignatura y su predisposición hacia el aprendizaje de esta materia.

Respecto a los contenidos, el ciclo se ha ajustado a la nueva estructura del tema en la nueva reorganización del temario. En este he incorporado algunos ejercicios para abordar las deficiencias detectadas en la evaluación inicial de conocimientos matemáticos previos, como son los errores de cálculo y razonamiento lógico. También he planteado las aplicaciones económicas desde un punto de vista amplio para mostrar de forma clara el proceso que permite transformar un problema económico en un problema matemático y viceversa.

El planteamiento metodológico para este ciclo parte del aprendizaje activo en grupo. En esta propuesta, es fundamental el aprendizaje basado en problemas apoyado en exposiciones que aclaren los errores comunes a la mayoría de los estudiantes o los conceptos o procesos que no se hayan entendido con suficiente claridad. Este modelo pedagógico se enfoca en que el alumnado tome las riendas del proceso de aprendizaje y asuma la responsabilidad que le corresponde. En este proceso de enseñanza, he asumido el papel de guía del mismo, adecuándolo a las demandas del alumnado y al desarrollo del aprendizaje.

La evaluación del aprendizaje ha estado basada en la evolución del mismo, tanto a nivel individual como grupal, 
proponiendo una evaluación formativa y continua. La evaluación se ha basado en la realización de tareas de aprendizaje-evaluación que permiten no solo evaluar y calificar, sino detectar errores para poder subsanarlos y adecuar la enseñanza a las verdaderas necesidades del estudiante y del grupo.

Para completar la evaluación, el alumnado ha respondido a un cuestionario de opinión sobre el modelo, seguido de un diálogo informal, grupal e individual, para conocer su percepción sobre el proceso y el desarrollo de este. De esta forma, el alumnado se implica en el proceso y me permite introducir las mejoras que demanden.

La valoración de los logros de aprendizaje se ha realizado de forma objetiva a través de las respuestas al cuestionario inicial-final.

\section{Mapa de contenidos}

El mapa de contenidos que se presenta para este ciclo se sitúa en torno a la necesidad y utilidad de las Matemáticas en conceptos económicos básicos, en particular de las funciones y de la derivada, y mostrar su aplicación.

Los contenidos que se abordan en el ciclo y las relaciones entre estos aparecen recogidos en la figura 1.

Son cuatro las preguntas que guían el mapa y que integran el cuestionario inicial-final con el que evaluar el conocimiento. En este caso, las preguntas del cuestionario están centradas en comprobar si el estudiante es capaz de relacionar conceptos económicos con conceptos matemáticos y comprende la aplicación de estos conceptos y herramientas matemáticas.

Jornadas de Formación e Innovación Docente del Profesorado | № 2 (2019) Esta obra se distribuye con la licencia Creative Commons Reconocimiento-NoComercial-SinObraDerivada 


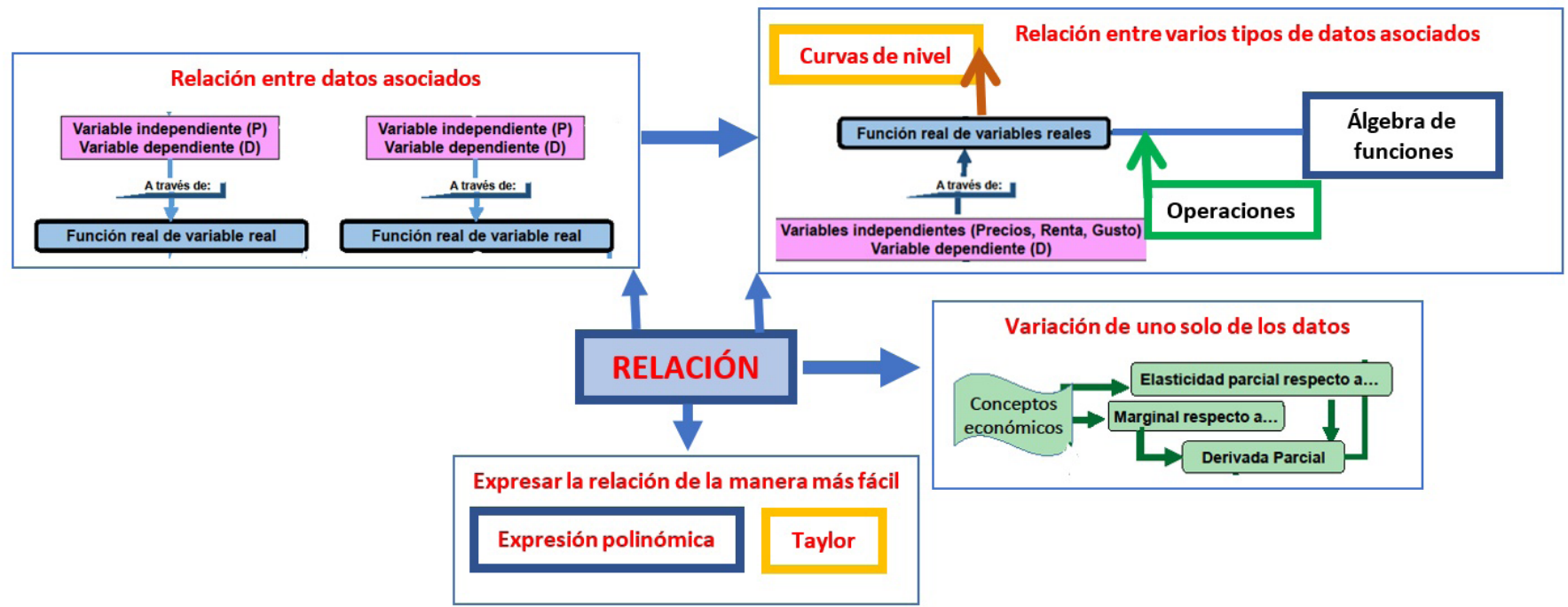

Figura 1. Mapa conceptual del Ciclo de Mejora en el Aula (CIMA)

Las preguntas del cuestionario inicial-final son las siguientes:

1. ¿Puede la demanda estar relacionada y condicionada por el precio? ¿Se puede expresar matemáticamente? Indique cómo.

2. Si la demanda de un bien es $D=D(P)$, ¿se puede calcular matemáticamente cómo afecta a la demanda la variación del precio en una unidad?

3. Si la demanda de un bien es $\mathrm{D}=\mathrm{D}(\mathrm{P})$, ¿se puede calcular cómo afecta a la demanda la variación del precio eliminando las unidades de medidas de ambos?

4. ¿Es posible una expresión más sencilla de la propia función? Indique cómo. 


\section{Modelo metodológico posible}

En este curso el planteamiento modelo metodológico inicial que he propuesto es flexible con el objetivo de poder dedicar más de tiempo a aquellas tareas o actividades propuestas en las que alumnado necesite más tiempo para terminarlas o simplemente porque surja algún aspecto que debatir o problema a resolver que creía asimilado o resuelto.

El modelo se configura en torno a tres bloques centrales (figura 2) que se abordan desde un perfil teórico y/o práctico (también se ha indicado el bloque de inicio de cada clase).

Para representar el modelo propuesto inicialmente he utilizado los siguientes elementos:

1. Cada parte en que se divide la clase está representada mediante una elipse. Su tamaño está en función del tiempo que se propone para dedicarle a éste.

2. En cada uno de los bloques anteriores aparecen las distintas tareas y el tiempo que se propone para dedicarles. Están representadas con un rectángulo que incluye el tiempo propuesto.

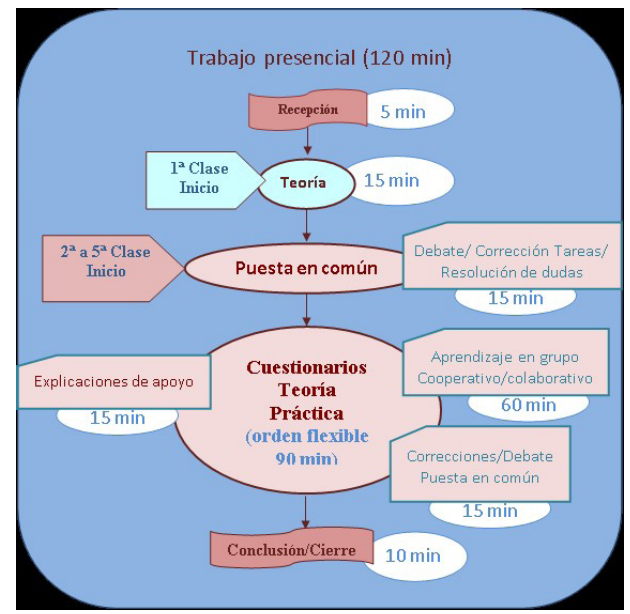

Figura 2. Modelo de trabajo en el aula

Jornadas de Formación e Innovación Docente del Profesorado | № 2 (2019) Esta obra se distribuye con la licencia Creative Commons Reconocimiento-NoComercial-SinObraDerivada 
La recepción y cierre de la clase es común durante las 5 sesiones del ciclo y tienen asignados 15 minutos en total.

En el primer bloque está indicada la organización de la parte inicial de la primera clase del ciclo. En ésta los estudiantes responden al cuestionario inicial, lo que me permitirá introducir el contenido matemático del tema relacionándolo con su aplicación económica. En las cuatro clases siguientes, este tiempo se dedica al planteamiento de dudas por parte del alumnado y a la resolución de las mismas en grupo fomentando la participación del alumnado. En el caso en que no se logre la aclaración de determinadas dudas, intervendré para ayudar.

La parte central de cada clase, 90 minutos, se dedican al trabajo en grupo resolviendo problemas, matemáticos y de tipo económico. Estos problemas servirán de repaso de los contenidos trabajados y para comprobar si hay que volver a incidir en ellos o ya han sido asimilados. También se introducirán algunos conceptos nuevos y se trabajará para sobre ellos en ejercicios y problemas que resolverán los estudiantes en la pizarra.

En este planteamiento son relevantes dos elementos básicos del aprendizaje en grupo cooperativo: la interacción cara a cara y la evaluación de los resultados del aprendizaje y del proceso (Johnson et al., 1999).

En dos de las cinco clases, segunda y cuarta, se propone inicialmente introducir cuestionarios como actividades de contraste, y en la última, clase el alumnado volverá a responder al cuestionario inicial.

Por último, el tiempo para cerrar la clase es de $10 \mathrm{mi}-$ nutos que dedicaré a indicar el trabajo no presencial de preparación de la próxima clase (lecturas, ejercicios, problemas, visionado de vídeos) y a hacer un pequeño resumen de los temas abordados. 
Siguiendo a De Miguel (2009), en este modelo, la planificación del escenario y los procedimientos se ha realizado en base a lograr que en el desarrollo del proceso de aprendizaje esté incluida la interacción y el feedback entre el grupo y el docente para alcanzar los fines de aprendizaje propuestos.

\section{Secuencia de actividades}

Las actividades propuestas para el trabajo que se realiza en el bloque central de la clase abordan la resolución de los ejercicios prácticos propuestos en la asignatura. Con estos ejercicios se persigue que el estudiante adquiera soltura en el cálculo del dominio de funciones, identificando la operación u operaciones del álgebra de funciones de las que proviene, el estudio de la continuidad de las funciones, el cálculo de las derivadas parciales y el cálculo de las aproximaciones polinómicas de funciones.

El resto de las actividades son problemas de enunciado económico propuestos en la asignatura y en los libros de la bibliografia recomendada. Estos problemas proponen el estudio de la marginalidad parcial y elasticidad, a través de la derivada parcial e interpretación de su signo y valor en términos económicos.

La secuencia de actividades está expuesta en la tabla siguiente.

Jornadas de Formación e Innovación Docente del Profesorado | № 2 (2019) Esta obra se distribuye con la licencia Creative Commons 
Tabla 1. Secuencia de actividades del Ciclo de Mejora en el Aula (CIMA)

\begin{tabular}{|c|l|l|}
\hline Clase & $\begin{array}{l}\text { Ejercicios prácticos/Problemas } \\
\text { Boletín de la asignatura }\end{array}$ & $\begin{array}{l}\text { Ejercicios propuestos } \\
\text { Boletín de la asignatura }\end{array}$ \\
\hline Primera & $\begin{array}{l}\text { a, b, c (dominios) } \\
\text { 3a, b (curvas de nivel) } \\
4 \text { (problema económico/ curva de } \\
\text { nivel) }\end{array}$ & $\begin{array}{l}1 \mathrm{~d}, 1 \mathrm{e} \\
3 \mathrm{c}, 3 \mathrm{~d} \\
5 \text { (problema económico/ curva de } \\
\text { nivel) }\end{array}$ \\
\hline Segunda & $\begin{array}{l}6 \mathrm{a}, \mathrm{b} \text { (álgebra de funciones) } \\
7 \mathrm{a}, \mathrm{b}, \mathrm{c} \text { (continuidad) }\end{array}$ & $\begin{array}{l}6 \mathrm{c} \\
7 \mathrm{~d}, \mathrm{e}, \mathrm{f}\end{array}$ \\
\hline Tercera & $\begin{array}{l}12 \text { (marginalidad, elasticidad) } \\
8 \mathrm{a}, \mathrm{b}, \mathrm{c}, \mathrm{d}, \mathrm{e}, \mathrm{f} \text { (derivadas parciales) }\end{array}$ & $\begin{array}{l}8 \mathrm{~g}, \mathrm{~h}, \mathrm{i}, \mathrm{j}, \mathrm{k} \\
13 \text { (marginalidad, elasticidad) }\end{array}$ \\
\hline Cuarta & $\begin{array}{l}14 \text { (marginalidad, elasticidad) } \\
9 \mathrm{a}, \mathrm{b} \text { (derivadas parciales segundas) } \\
15 \mathrm{a}, \mathrm{b}, \text { (polinomio de Taylor) }\end{array}$ & $\begin{array}{l}9 \mathrm{c}, \mathrm{d}, \mathrm{e}, \\
15 \mathrm{a}, \mathrm{b} \text { (polinomio de Taylor) }\end{array}$ \\
\hline Quinta & $\begin{array}{l}16 \mathrm{a}, \mathrm{b} \text { (desarrollo de Taylor) } \\
17 \mathrm{a}, \mathrm{b}, \text { (desarrollo de McLaurin) }\end{array}$ & $\begin{array}{l}\text { Propuesta: terminar los 18 ejercicios } \\
\text { del tema. }\end{array}$ \\
\hline
\end{tabular}

Durante la realización de los ejercicios de la columna de la izquierda, promuevo en clase un debate/corrección de los ejercicios que aparecen en la columna derecha.

Esta planificación está enfocada en la reflexión sobre el propio aprendizaje del estudiante para que identifique su forma de aprender (metacognición) y sea capaz de elegir y aplicar las herramientas matemáticas, en un proceso en el que aprende ayudado por el grupo y el docente. Según Moreno (2006), la mayoría de las teorías coinciden en que estas son las bases necesarias para lograr aprender a aprender.

En este planteamiento inicial del ciclo, la propuesta incluye dos cuestionarios de respuesta múltiple realizados on line como pruebas de control. Esta opción permite dinamizar el aula y generar debate y un proceso de reflexión sobre las respuestas. A partir de sus resultados se pueden identificar las deficiencias y fortalezas del aprendizaje 
que son comunes a la mayoría de los estudiantes. Estas pruebas también se orientan hacia la autoevaluación del alumnado ya que, a través de sus preguntas, el estudiante puede conocer aquello que ha logrado aprender y lo que no (Castillo y Cabrerizo, 2010).

La primera prueba está programada al iniciar la segunda clase y la segunda al comienzo de la cuarta clase.

\section{Aplicación del Ciclo de Mejora en el Aula}

Para analizar los resultados del ciclo voy a tomar los datos referentes a los 31 estudiantes que han asistido a todas las clases del ciclo, es decir, los que lo han completado.

El cuestionario de conocimientos básicos se realizó el día de la presentación de la asignatura y proporcionó información sobre el nivel de conocimiento matemático previo en relación con el tema de las funciones reales de variable real y del manejo de conceptos matemáticos básicos como la derivada.

A continuación, se exponen aquellos aspectos relevantes en el desarrollo del ciclo en el aula, tanto los que se han mantenido en la práctica como los nuevos que se han introducido y que no aparecían recogidos en el planteamiento anterior.

El primer día del ciclo, los estudiantes han respondido al cuestionario inicial que aparece en el apartado anterior.

Se ha mantenido la metodología propuesta, aprendizaje activo en grupos colaborativos/cooperativos basado en problemas, durante la parte central de la programación de cada clase. El aula es un escenario que ha favorecido que el trabajo se desarrolle en grupo y que haya habido interacción estudiante-grupo-docente, implicándose el 
alumnado en el proceso aprendizaje. En los grupos se ha debatido y razonado sobre los pasos de la resolución de los diferentes ejercicios y problemas contextualizados.

Durante el trabajo en grupos he supervisado el desarrollo de la resolución de los problemas que he propuesto y tomado nota de las dificultades que han ido apareciendo en los diferentes grupos. Si estas han sido generalizadas, he realizado una pausa en el trabajo para intervenir y explicar cómo abordar la parte problemática. Si las dificultades se localizan en un grupo determinado, entonces he iniciado un diálogo con sus integrantes para intentar deducir con estos los pasos a seguir para resolver el problema.

La propuesta de trabajo no presencial se ha desarrollado como estaba prevista, basada en la lectura de cada parte del tema que se ha abordado en clase y en los ejercicios del boletín que sobre los conceptos explicados en el aula para poder ser corregidos en las siguientes clases. Estos ejercicios tienen distintos niveles de dificultad.

Para comprobar el trabajo y desarrollo del aprendizaje, tanto dentro como fuera del aula, se han desarrollado las dos actividades de control previstas. Estas han sido corregidas en el aula para que el alumnado pueda comprobar lo que responde correctamente y afianzarlo, y lo que necesita trabajar. De esta forma se ha producido un feedback inmediato que ha enriquecido el proceso.

Un aspecto importante de la información que me han proporcionado estas dos pruebas es que me ha permitido introducir cambios en las sesiones de trabajo presencial para corregir las deficiencias detectadas en el aprendizaje de los diferentes contenidos. Así, he necesitado introducir en clase una serie de tareas que no estaban previstas para que el alumnado mejore su comprensión y manejo del cálculo de derivadas y la interpretación de sus resultados en problemas económicos. Esto ha provocado la modificación de la planificación expuesta anteriormente. Como consecuencia no se ha podido abordar en el ciclo la última parte dedicada a la aproximación de funciones. 
A partir de esta reorganización del contenido del ciclo, en el cuestionario final se ha eliminado la última pregunta relacionada con la aproximación de funciones, manteniéndose las otras tres. El alumnado ha respondido a estas cuestiones al final de la quinta clase.

Este proceso de evaluación a través de los cuestionarios y las pruebas de control ha supuesto un "un proceso dinámico, abierto y contextualizado" (Castillo y Cabrerizo, 2010, p. 17) que ha proporcionado información con dos instrumentos de evaluación diferentes, cuyo análisis me ha permitido tomar decisiones para reorganizar el aprendizaje.

Para analizar los resultados del cuestionario inicial-final, he establecido unos niveles en los que agrupar las respuestas a cada una de las tres preguntas. En la primera pregunta he establecido siete niveles, los dos primeros, "No sabe/ No contesta" y "Sí/No", están asociados a la ausencia de razonamiento o reflexión en torno a la pregunta. El tercer y cuarto nivel recogen respuestas exclusivamente en términos económicos, situando en el primero de ellos las respuestas incorrectas. Si la respuesta se realiza en términos matemáticos es incluida en el quinto nivel, y en el sexto si se relacionan con los conceptos económicos. Por último, hay respuestas que introducen relaciones más amplias y que se recogen en el nivel 7.

En la segunda y tercera pregunta, también partimos de estos siete niveles. Para la pregunta 2 se ha eliminado el último nivel y para la pregunta 3, los tres últimos. De esta forma, la pregunta 2 tiene sus respuestas organizadas en seis niveles y la pregunta 3 en cuatro niveles.

La figura 3 recoge la escalera de aprendizaje de la primera pregunta y los porcentajes de respuestas de cada nivel, tanto en el cuestionario inicial como en el final (enmarcado en un rectángulo). Las figuras 4 y 5 recogen estos mismos datos para la pregunta 2 y 3 , respectivamente. 


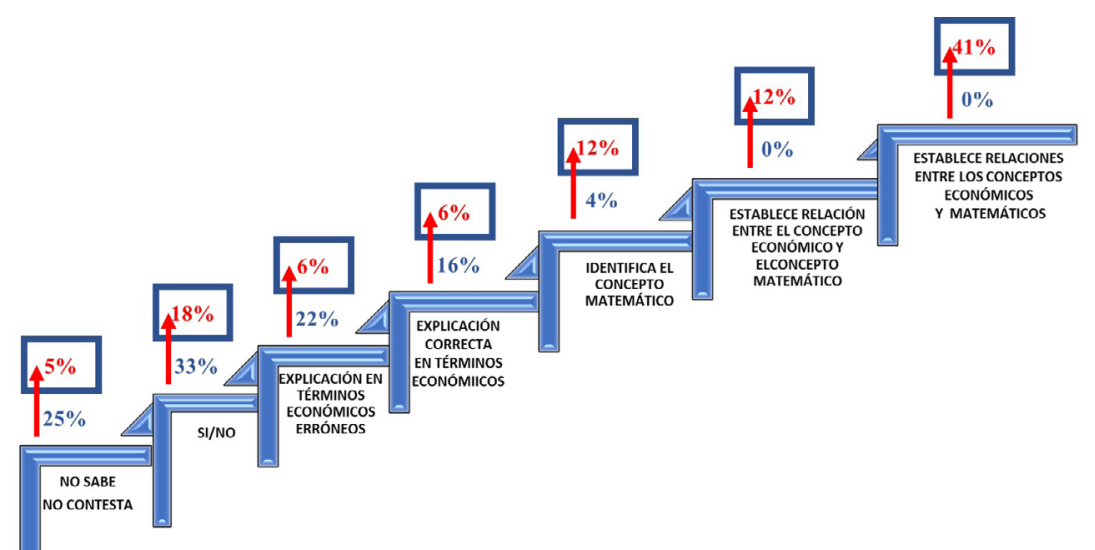

Figura 3. Escalera pregunta 1

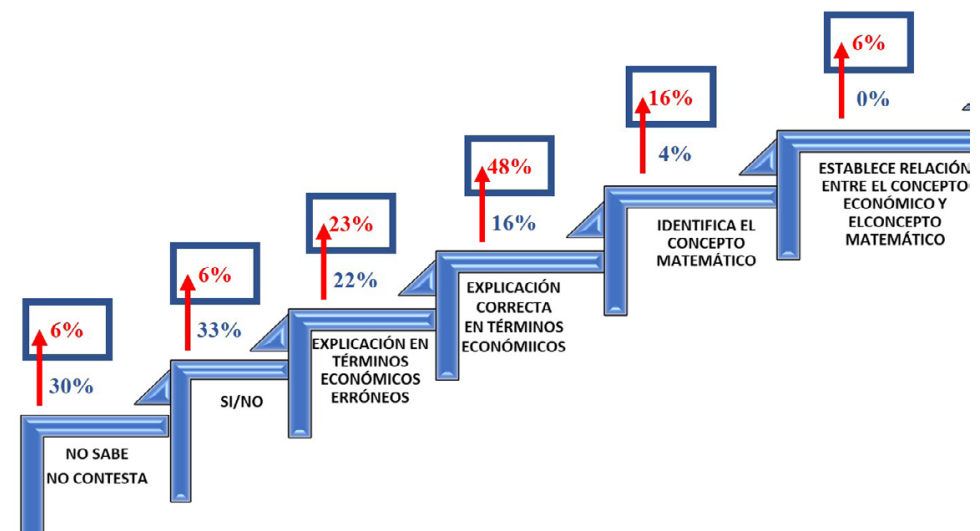

Figura 4. Escalera pregunta 2

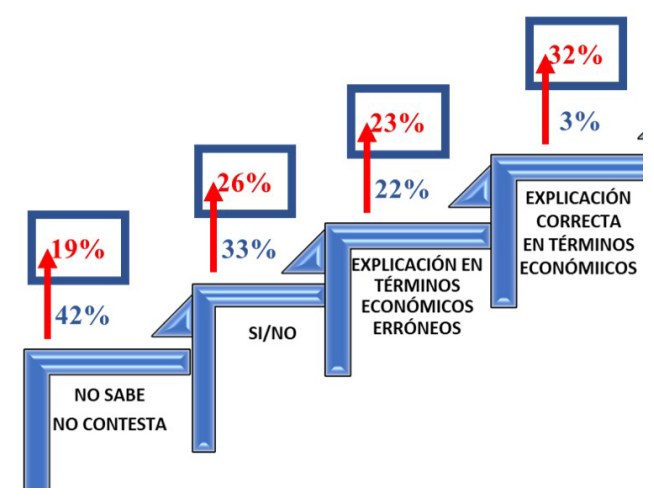

Figura 5. Escalera pregunta 3

Jornadas de Formación e Innovación Docente del Profesorado | № 2 (2019) Esta obra se distribuye con la licencia Creative Commons Reconocimiento-NoComercial-SinObraDerivada Internacional (CC BY-NC-ND 4.0.) 
Los porcentajes de las respuestas de las preguntas correspondientes al cuestionario inicial recogidos en las tres escaleras anteriores muestran una concentración importante de respuestas, entre el $58 \%$ y el $75 \%$, en los dos niveles inferiores. También se observa, independientemente de la pregunta, una ausencia de respuestas en los niveles superiores que implican un razonamiento sobre la relación entre los conceptos matemáticos y económicos. Esto se debe a que la aplicación de las matemáticas en el ámbito económico es nueva para el alumnado como lo es el contenido matemático del ciclo en torno a la función real de varias variables reales.

Los resultados del cuestionario final muestran que el alumnado ha avanzado en el conocimiento sobre la aplicación de conceptos matemáticos en el estudio y cálculo de conceptos económicos, en este caso en la marginalidad y la elasticidad. Estos resultados evidencian que se ha conseguido avanzar en el desarrollo del aprendizaje y que ha sido adecuado dedicar dos horas más a la enseñanza de estos contenidos.

En las tres escaleras se observa un descenso de respuestas en tres niveles inferiores, lo que se interpreta de manera positiva para el aprendizaje. Sin embargo, los porcentajes de los niveles superiores muestran que aún es necesario insistir en las aplicaciones económicas de la derivada, en particular en el significado del concepto de elasticidad y su cálculo.

Jornadas de Formación e Innovación Docente del Profesorado | № 2 (2019) Esta obra se distribuye con la licencia Creative Commons 


\section{Evaluación del ciclo de mejora}

Durante el desarrollo del ciclo he elaborado un diario de clase en el que he recogido el desarrollo de cada clase, especificando las actividades realizadas (previstas en el diseño previo y aquellas incluidas como refuerzo), los resultados de las actividades de control, las deficiencias detectadas y los objetivos alcanzados.

Salvo el reajuste en el tiempo dedicado a los contenidos, eliminando la última parte de estos dedicado a la aproximación de funciones, se ha seguido la planificación programada previamente. Este hecho lo tendré en cuenta a la hora de elaborar la programación del próximo curso. También incorporaré ejercicios y problemas relacionados con los puntos más problemáticos que han sido identificados durante el desarrollo del CIMA de este curso.

En el ciclo he intentado que el clima de clase fuera adecuado para poder interactuar con el alumnado y favorecer un diálogo que me ayudara a comprobar si se estaba produciendo un avance en el aprendizaje o si era necesario reajustarlo, como así ha ocurrido.

En este diálogo, he hecho participe al alumnado en la decisión del tipo de ejercicios y problemas sobre los que ha sido necesario insistir, favoreciendo su capacidad para identificar sus fortalezas y debilidades de aprendizaje.

Por último, he pedio al alumnado su opinión sobre sobre la experiencia y la dinámica de las clases, siendo positiva la percepción que tienen sobre esto. Sin embargo, hay que señalar que el alumnado se siente agobiado e inseguro durante el primer cuatrimestre de sus estudios, ya que estudiar en la universidad supone un enorme cambio, tanto por el contenido como por la intensidad de las clases. Esto supone un enorme esfuerzo de adaptación. 
En el próximo curso me planteo incorporar más pruebas de control durante el ciclo ya que me han permitido reajustar la planificación.

Personalmente, esta experiencia me ha permitido ampliar mi visión sobre la enseñanza de los conceptos de este tema y las posibilidades de proponer su aprendizaje desde su justificación y necesidad dentro de este tipo de estudios. Si se mantiene el temario, en el próximo curso me planteo ampliar el ciclo al contenido que no he podido abordar en éste. 


\section{Referencias bibliográficas.}

Castillo, S. y Cabrerizo, J. (2010). Evaluación educativa de aprendizajes y competencias. Madrid: Pearson.

De Miguel, M. (Dir.) (2009). Metodologías de enseñanza y aprendizaje para el desarrollo de competencias. Orientaciones para el profesorado universitario ante el Espacio Europeo de Educación Superior. Madrid: Alianza.

Moreno, M. M. (2006). Un camino para aprender a aprender. México: Trilla.

Jornadas de Formación e Innovación Docente del Profesorado | № 2 (2019) Esta obra se distribuye con la licencia Creative Commons 